\title{
Wegener's granulomatosis: surgical repair of severe subglottic stenosis
}

\begin{abstract}
Laryngeal and subglottic stenosis are present in about $20 \%$ of patients with granulomatosis with polyangiitis. This atypical form of the disease can be severe and life-threatening. In what concerns to treatment, subglottic lesions are less responsive to conventional systemic therapy and interventional and surgical procedures should be considered. The authors present a case of a 30-year-old woman with diagnosis of Wegener's granulomatosis who presented to our department with dyspnea and stridor due to a severe subglottic stenosis, requiring an emergent tracheostomy. The obstructive defect was electively and effectively repaired through a transoral laser microsurgery using local application prednisolone and mitomycin $\mathrm{C}$ followed by balloon dilation.
\end{abstract}

Keywords: acquired subglottic stenosis, chronic granulomatous disease, wegener granulomatosis, airway management, reconstructive surgery
Volume I2 Issue 4 - 2020

\begin{abstract}
Isabel Costa,António Lima, Cátia Azevedo, Fernando Mar, Berta Rodrigues, Luís Dias Ear Nose and Throat and Head and Neck Surgery department, Hospital de Braga, Portugal
\end{abstract}

\begin{abstract}
Correspondence: Isabel Costa, Ear Nose and Throat and Head and Neck Surgery department, Hospital de Braga, Portugal, Rua Sete Fontes, São Vitor - Braga, Portugal, Tel +35 19| I058438, Email isabelcostorl@gmail.com
\end{abstract}

Received: June 25, 2020 | Published: July 16, 2020
Abbreviations: ANCA, anti-neutrophil cytoplasmic antibody; GPA, granulomatosis with polyangiitis; MRI, magnetic resonance imaging, SGS, subglottic stenosis

\section{Introduction}

Subglottic stenosis (SGS) is present in about $12-23 \%$ of patients with granulomatosis with polyangiitis (GPA, also known as Wegener's granulomatosis). This situation is reported to be more common among children and young adults. Macroscopically, SGS caused by Wegener's granulomatosis appears as a circular, reddish and friable narrowing below the vocal cords. ${ }^{1-3}$ In what concerns to treatment, subglottic lesions are less responsive to conventional systemic therapy, which may actually favor local fibrosis. Therefore, systemic agents may not be primarily indicated in isolated SGS and interventional procedures should be considered. ${ }^{4}$

\section{Case report}

The authors present a case of a 30-year-old woman who use to work as a lyric singer, with a history of chronic otorrhea and recurrent otalgia associated with chronic nasal obstruction, relapsing epistaxis and headaches. Ear examination showed a double perforation on the left tympanic membrane and the nasopharyngolaryngoscopic examination showed bilateral ulceration and crusting of the nasal mucosa, without any laryngeal findings. Nasal and pulmonary biopsies showed a granulomatous and necrotic inflammatory process, compatible with granulomatosis with polyangiitis (GPA). Anti-neutrophil cytoplasmic antibody (ANCA) levels were elevated. Imaging study revealed bilateral chronic rhinosinusitis and a pulmonary nodule (Figure 1). She was then treated with prednisolone and azathioprine. A year later, after the negativity of ANCA levels, systemic improvement and initiation of immunosuppressors withdrawal, she was admitted to the emergency department with dyspnea and stridor, requiring an emergent tracheostomy. At that time, the laryngological examination showed a severe subglottic stenosis with a maximum diameter of $4 \mathrm{~mm}$ (Figure 2A). One week later, she underwent elective surgical repair of the subglottic defect with laser, balloon dilation, and mitomycin and prednisolone local application. First, endoluminal laser star incisions were performed so we can enlarge the airway lumen (Figure 2B). Next, we proceed to local infiltration of prednisolone and application of mitomycin $\mathrm{C}$ followed by dilation with an $11 \mathrm{~mm}$ balloon and hemostasis (Figure 2C). After 2 years of follow-up, there are no signs of tracheal stenosis. She started singing again.
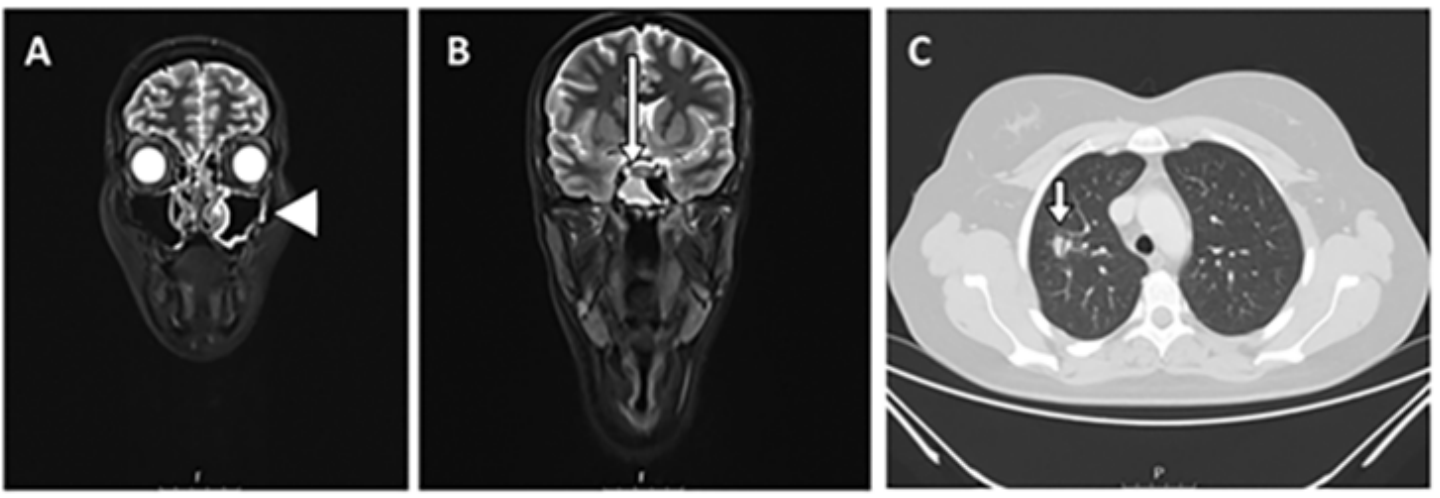

Figure I Imaging study with maxillo-facial and neck magnetic resonance imaging (MRI) revealed signs of chronic rhinosinusitis with bilateral inflammatory mucosal thickening of ethmoid, maxillary and sphenoid sinuses (arrowhead), particularly in the right sphenoid sinus (long arrow) (A, B). MRI of the lung showed the presence of an irregular nodule in the right superior lobe $(17 \mathrm{~mm})$ and another adjacent regular nodule with II mm (C, short arrow). 


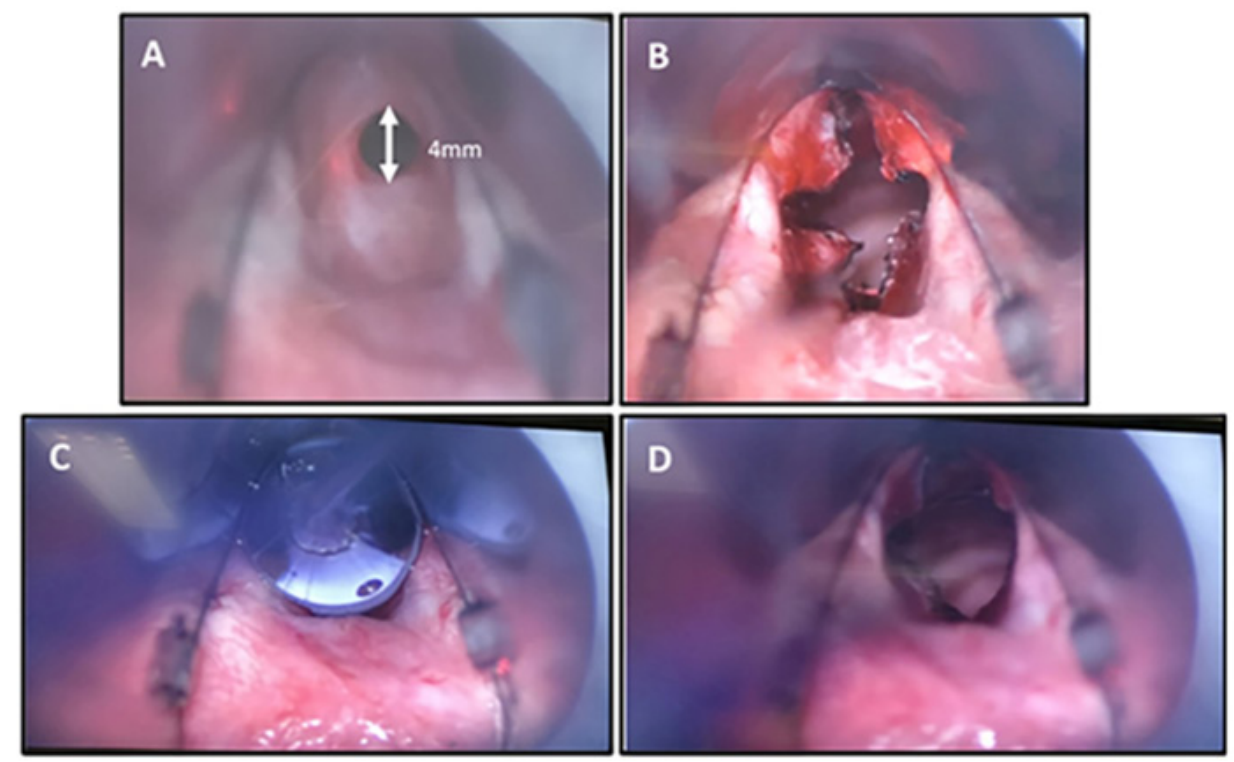

Figure 2 Transoral laser microsurgery under general anesthesia: introduction of the laryngoscope and visualization of subglottic stenosis with a maximum diameter of $4 \mathrm{~mm}$ (A). Endoluminal laser star incisions to enlarge the airway lumen (B). Next, we performed local infiltration of prednisolone and application of mitomycin $\mathrm{C}$ followed by dilation with an $1 \mathrm{Imm}$ balloon and hemostasis (C). Final result (D).

\section{Discussion}

Here we reported a case of an aggressive granulomatosis with polyangiitis with rhino-sino-auricular and laryngeal tropism. Otorhinolaryngological GPA's manifestations are common but subglottic stenosis and tracheal stenosis are less common. The first one is present in about 10-20 per cent of cases and may occur either as a presenting feature or as late-stage manifestation of the disease. ${ }^{1,2}$ SGS is considered a localized form of GPA and this form of disease is expected to be ANCA-negative and does not correlate with active stages of disease. In this case, and similarly to what reports suggest, SGS occurs or progresses independently of other features of active disease, and sometimes appears while the general disease is in remission. ${ }^{4}$ The subglottic lesion is usually atypical and relapsing because it is frequently refractory to systemic and conventional immunosuppressive therapy. Aggressive treatment may also lead to local fibrosis., ${ }^{3,4}$ For all these reasons, systemic agents may not be indicated in isolated SGS and interventional procedures (such as the one that we described here) should be considered.

\section{Conclusion}

The authors highlight the importance of close monitoring of patients with laryngeal and tracheal GPA, even in stages of apparent remission of the disease. The authors conclude that certain surgical approaches can be feasible and effectives treatment options for patients with symptomatic stenotic of the subglottis and trachea.

\section{Acknowledgments}

None.

\section{Conflicts of interest}

None of the authors declares conflicts of interest.

\section{Funding}

None.

\section{References}

1. Gluth MB, Shinners PA, Kasperbauer JL. Subglottic stenosis associated with Wegener's granulomatosis. Laryngoscope. 2003;113(8):1304-1307.

2. Erickson VR, Hwang PH. Wegener's granulomatosis: current trends in diagnosis and management. Curr Opin Otolaryngol Head Neck Surg. 2007;15(3):170-176.

3. Rasmussen N. L24. Local treatments of subglottic and tracheal stenosis in granulomatosis with polyangiitis (Wegener's). Presse Med. 2013;42(4 Pt 2):571-574.

4. Solans Laqué. Clinical characteristics and treatment of subglottic stenosis in patients with Wegener's granulomatosis. Int. J. Clin. Rheumatol. 2010;5(4):475-486. 